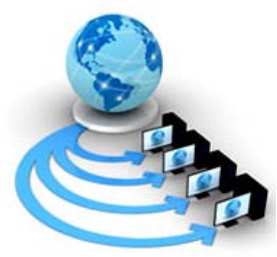

Volume 8, No. 7, July - August 2017

\title{
TRUSTWORTHINESS OF THE USERS AND PRODUCTS BASED ONUSER REVIEWS ON ECOMMERCE SITES
}

\author{
Radhika Patwardhan ${ }^{1}$, Prof.Narendra Gawai ${ }^{2}$ \\ ${ }^{1}$ MTech CST Student ${ }^{2}$,Asst. Professor, \\ ${ }^{1,2}$ CST DEPT ,UMIT, SNDT University, \\ Mumbai India
}

\begin{abstract}
Internet has become one of the most important part of everyone's life. There are billions and billions of internet users increasing every day. Shopping with e-commerce sites has also become an integral part of our routine because it has many advantages over the traditional shopping such as time consumption and large variety of options available on a single click. Even though no reasonable person will spend money at an untrustworthy online store no matter how usable and pretty the site may be. Trust in online site strongly affects consumers' purchase decisions. These decisions are mainly dependent on the reviews and findings written by the customers. In this paper we solely focus on the trust factors of the reviews written by the users on each product. We then calculate the trust factor of the product based on the calculated trust value. The first section includes related work in this area. In the following sections we discuss the detailed algorithms, modules and some implementation techniques. The last section discusses conclusion and future scope for the problem.
\end{abstract}

Keywords :-Concordance, opinion mining, Polarity, TRS

\section{INTRODUCTION}

Business in E-commerce is spreading its wings rapidly over the traditional business worldwide. The benefits of ecommerce, ease and comfort for the buyers, secured transactions are few important reasons behind the things being so successful.

It's a very common practice to provide feedbacks / ratings on the products purchased online by the buyers. The analysis of trustworthiness of such feedbacks/ ratings is the main issue of concern.

Trust is an important factor in any social relationship and especially in commerce transactions. In traditional commerce, a buyer can often see both the seller and the product, verify its quality, negotiate and bargain with the seller. Thus, it is possible for each of them to assess the trustworthiness of the other and for the buyer to be convinced about the product goodness. In the e-commerce context, there is a lack of direct trust assessment.

E- Commerce does not have any direct assessment of trust to it, so the users refer to the reviews of the products, in order to conceive their own trust and reputation experience. By this reviews of the product it trusts the product and purchases it, Therefore feedbacks overviews, scores, recommendations and any other information given by users are very important for the trust reputation assessment. However the reliability of the reviews must be verified to ensure only genuine review is taken into consideration. Here lies the importance of Trust Reputation System (TRS) implementation because this is the need of an hour for the sellers, production companies as well as buyers.

In this paper we focus on the related work in this area in the next section. The proposed system will be introduced in the further section which will be followed by implementation section. The paper concludes with future work and conclusion.

\section{RELATED WORK}

Hasnae RAHIMI, Hanan EL BAKKALI [1] at al, define the trust as the ability to rely on someone or something, to rely on its truthiness, on its strength to prove its reliability. In ecommerce, being trustful is a quality characterizing a product that a user claims to know either intuitively or from a past experience which is more trustful, or because other users estimate that it is a reliable product. They define TRS as - Trust and reputation systems (TRS) are an important class of decision support tools that can help reduce risk when dealing with in transactions and interactions online.

The authors provide in this paper is a Reputation algorithm which studies the user's attitude toward this selection of prefabricated feedbacks. As a result of this study, the reputation algorithm generates better trust degree of the user, trust degree of the feedback and a better global reputation score of the product.

The paper gives the basic idea of trustworthiness and how it is measured in E Commerce site, it also explains the use of prefabricated feedback to give the user a trust degree and products its global reputation score. With the help of these two trust degree and the user appreciation about the productthe products global reputation score is calculated and even the trust degree of the feedback given by the user.

Xiuzhen Zhang, Lishan Cui [2] suggest in their paper the idea of calculation of trust factors considering the seller's on ecommerce web sites. They try to evaluate and find solution to the famous "all good reputation" problem. 
They propose a model CommTrust for trust evaluation by mining feedback comments. The main contributions include: 1) a multidimensional trust model for computing reputation scores from user feedback comments; and 2) an algorithm for mining feedback comments for dimension ratings and weights, combining techniques of natural language processing, opinion mining, and topic modelling, They have developed a Comment-based Multi-dimensional trust (CommTrust), a fine-grained multi-dimensional trust evaluation model by mining e-commerce feedback comments. With CommTrust, comprehensive trust profiles are computed for sellers, including dimension reputation scores and weights, as well as overall trust scores by aggregating dimension reputation scores. Their research has helped in reducing "all good reputation" problem and rank sellers effectively on the live sites like eBay and Amazon.

Lifeng Wang [3] at al, propose a novel trustworthiness measurement model, which has the capability to deal with all types of possible trust factors leading to a final trustworthiness decision making. The evaluation of trustworthiness is based on our designed multi-criteria analysis mechanism. They developed a system which recognizes trust factors of e-commerce systems and distributes the factors in the designed multi-dimensional trust space and trustworthiness measurement model. All relevant factors are filtered, categorized and quantified. Then, the designed multi-criteria analysis mechanism can deal with the trust factors and analyze their trust features from different perspectives.

Hiral Y. Modi[4] introduces an effective algorithm for web mining which is going to be helpful for our algorithm for calculating trust factors while storing and analysing user's reviews on different product categories In this paper an architecture that integrates product information with user's access log data and then generates a set of recommendations for that particular user is presented. The implementation has recorded encouraging results in terms of precision, recall and F1 metrics.

\section{III .ALGORITHMS AND MODULES}

The proposed system mainly intends to deal with the problem of Opinion mining and trustworthiness by handling the opinions by passing it to Trust reputation system (TRS). The TRS algorithm mainly tries to understand the user behaviour by evaluating trust values depending on the prefabricated feedbacks. We will discuss the modules in detail-

The project is roughly divided into three modules.

1. Website design

2. Feedback acceptance with opinion mining.

3. TRS algorithm for calculation of User and Product Trust.
The flow of the project is described in fig 1.

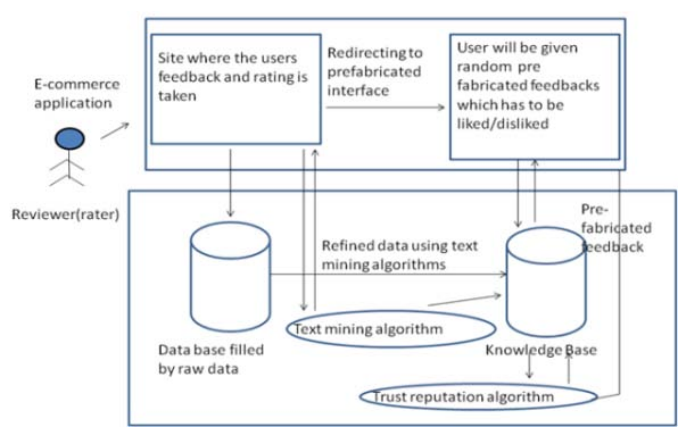

Fig 1. Flow diagram

a) Algorithm for Concordance Checking-

1. Accept the textual Feedback from User.

2. Perform Lexical Analysis on the text.

3. Use POS (part of speech) Tagging to separate the meaningful words.

4. POS tagging processes the marking up of a word in a text body as corresponding to a particular part of speech, based on both its definition and its context. i.e., its relationship with adjacent and related words in a phrase, sentence, or paragraph that marks the words in the review statement as noun, verb, adjective, adverb etc.

5. Process the marked words to identify nouns and adjectives, also adverbs associated with the nouns.

6. Compute the value of score of user's feedback using 'SentiWordNet' (a lexical mining resource) which gives a positive, negative or neutral feedback.(positive or negative polarity)

7. Accept the rating between the range of -5 to +5 from the user.

8. Compare the user rating[5] with the score calculated by lexical analysis, If it matches, accept the review and update the database with the latest concordance value.

9. Else Block the user for giving contradictory review and ratings and discard the review.

b) Algorithm for Trust Reputation System (TRS):-

1. Prepare a knowledge database with previously stored feedbacks of different users along with their trust values.

2. Select the reviews randomly from the knowledge base (pre-fabricated feedbacks) and display them before the user as a special form.

3. The user will agree or disagree the other user's reviews by clicking on radio buttons.

4. The Values entered by the user will be compared with the original trust values of the reviews and the trust factor of the user as well as latest review will be calculated.

5. The knowledge base and feedback table are updated.

6. A Global Trust Value for the Product will be updated and displayed on the sitewith the following formula:-

Global trust value $=\left(\mathrm{X}+\mathrm{Y}^{*} \mathrm{~b} / \mathrm{a}+\mathrm{b}\right)$

Where

$\mathrm{X}$ - stored trust value in the database

$\mathrm{Y}$ - recently calculated trust value

a-Summation on coefficient on feedbacks

b- current User ratings [5] 


\section{IMPLEMENTATIONDETAILS}

ASP .Net is used to develop the website whereas the algorithms are developed using C\# technology. The backend is developed with SQL server. The execution starts with the website where you can see the variety of products with all the details, which can be selected for purchase.

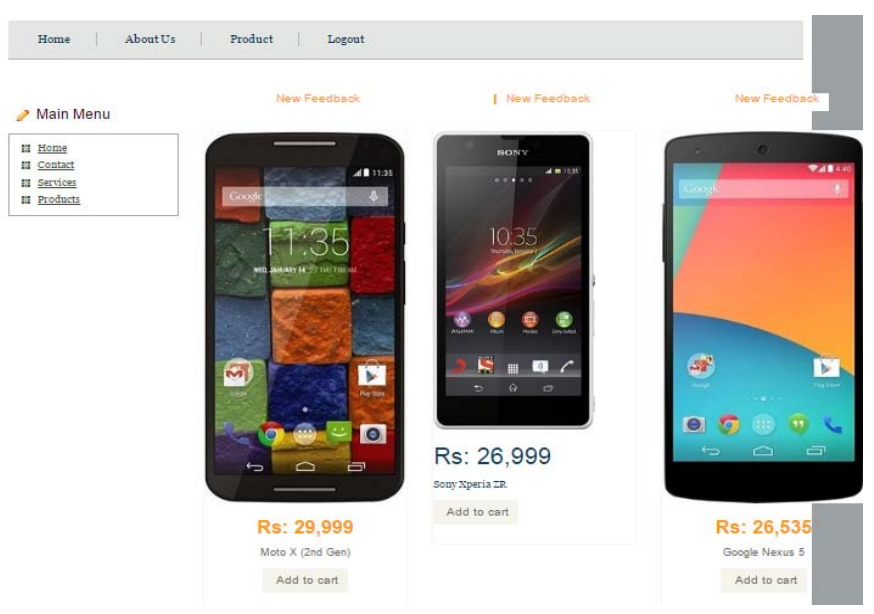

Fig. 2 Product catalogue

The selected products will be inserted in the cart, Here we can make the transactions and buy the product. The reviews can only be written if the product is purchased. The users can see the related reviews with their trust values on the website.

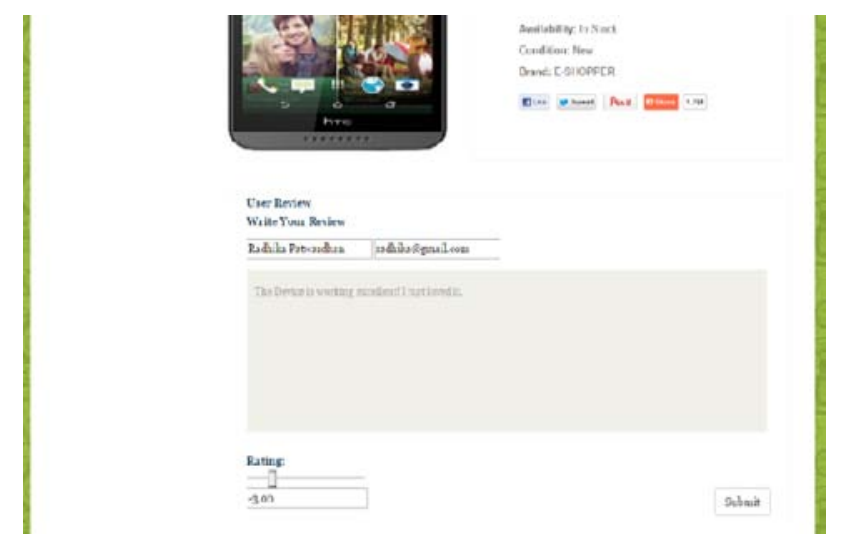

Fig. 3 .Writing a review with rating.

When the user writes the review and clicks the submit button a form with some random questions is displayed.
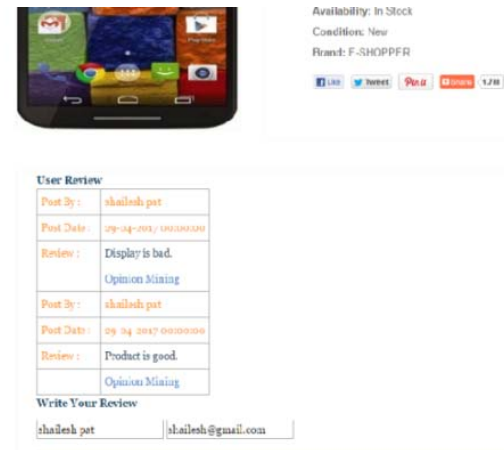

Fig. 4. Result of opinion mining.

The questions are selected randomly from the reviews stored in the database. The user selects the appropriate options and from these options the TRS algorithm calculates the results. If the results are positive, the user is considered to be trustworthy else he is banned from writing the reviews on the site for a random time. The more trustworthy reviews for the product, the more Trustworthy the product is. The global Trust value of the product is updated each time a new review is written.

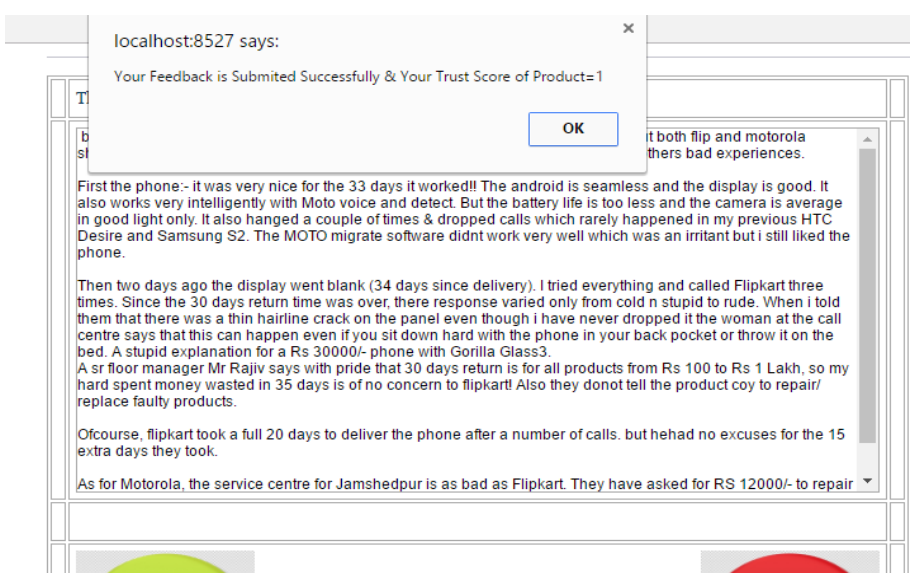

Fig. 5. Final Trust Value

This trust value will be stored in the database so that it can be used for future calculations on trust factors.

\section{CONCLUSION AND FUTURE WORK :-}

This way, the results of opinion mining, the polarity values, current feedback written by the user al0ng with the ratings, and a sum of already stored trust value for the particular product play a major role in finding a final TRS value for the product as well as user.

The more trustworthy feedbacks and products on the sites, the more customers will get attracted resulting in a good business.

AS a future work, the site can have a different types of analysis of reviews depending on the specifications of the products.

TRS is going to prove extremely useful for the users interested in purchasing various products from commercial websites. It will certainly help to detect the malicious users and fake reviews, so that users get benefit of purchasing 
only trustworthy products, which will enhance the site's business and reputation.

\section{REFERENCES}

[1]. Hasnae RAHIMI and Hanan EL BAKKALI ," A New Reputation Algorithm for Evaluating Trustworthiness in ECommerce Context”, Information Security Research Team (ISeRT), University Mohamed V-Souissi,Published in Security Days (JNS3), 2013 National, Conf. date 26-27 April 2013, ENSIASRABAT, Morocco,

[2]. .Xiuzhen Zhang, Lishan Cui, and Yan Wang ,"CommTrust: Computing Multi-Dimensional Trust by Mining E-Commerce Feedback Comments", IEEE TRANSACTIONS ON KNOWLEDGE AND DATA ENGINEERING, VOL. 26, NO. 7, JULY 2014, Pages: 1631 - 1643
[3]. .Lifeng Wang, "Evaluation of E-commerce System Trustworthiness Using Multi-criteria Analysis”,Department of Computer Science and Engineering, University of BridgeportBridgeport, CT 06604, USA, Published in: Computational Intelligence in Multi-Criteria DecisionMaking (MCDM), 2014 IEEE Symposium, Date of Conference: 9-12 Dec. 2014

[4]. Hiralmodi, "Enhancement of online web recommendation system using a hybrid clustering and pattern matching approach “ D.J. Sanghavi COE, Mumbai.

[5]. Published in: Nascent Technologies in the Engineering Field (ICNTE), 2015 International Conference,

[6]. Date of Conference: 9-10 Jan. 2015

[7]. Guoshuai Zhao, XuemingQian, "Service Quality Evaluation by ExploringSocial Users' Contextual Information”, IEEE TRANSACTIONS ON KNOWLEDGE AND DATA

ENGINEERING 2016, Volume: 28, Issue: 12, Dec. 12016 , Page: 3382 - 3394,Date of Publication: 08 September 2016 